\title{
Model order reduction of parameterized systems (MoRePaS)
}

\section{Preface to the special issue of advances in computational mathematics}

\author{
Peter Benner ${ }^{1}$ - Mario Ohlberger ${ }^{2}$. \\ Anthony T. Patera ${ }^{3}$. Gianluigi Rozza ${ }^{4}$. \\ Danny C. Sorensen ${ }^{5}$ Karsten Urban ${ }^{6}$ \\ Published online: 14 November 2015 \\ (C) Springer Science+Business Media New York 2015
}

Even though capacity of modern computers keeps growing, it has become evident over the past decades that the complexity of practically relevant problems from science and engineering has grown even faster. Without highly efficient, reliable and robust numerical methods there will be no progress in many areas. The complexity of relevant problems in turn has lead to systems of equations with an extremely large

Communicated by: Editors of Special Issue on MoRePas

Karsten Urban

karsten.urban@uni-ulm.de

Peter Benner

benner@mpi-magdeburg.mpg.de

Mario Ohlberger

mario.ohlberger@uni-muenster.de

Anthony T. Patera

patera@mit.edu

Gianluigi Rozza

grozza@sissa.it

Danny C. Sorensen

sorensen@rice.edu

1 Max Planck Institute for Dynamics of Complex Technical Systems, Sandtorstr. 1., 39106 Magdeburg, Germany

2 Institute for Computational and Applied Mathematics, Universität Münster, Einsteinstr. 62, 48149 Münster, Germany

3 Massachusetts Institute of Technology, 77 Massachusetts Avenue, Cambridge, MA 02139-4307, USA 
number of unknowns that need to be handled numerically. Without a significant reduction of the dimension of such systems accompanied with a mathematical analysis of accuracy, efficiency and robustness, several real-world problems will be out of reach also within the near future. This is exactly where model order reduction comes into play.

Model order reduction techniques have already been developed and used since quite some time. Quite often, such schemes have been derived solely from the properties of the specific real-world problem. However, it has become evident that a deep understanding of the mathematical foundation is necessary, on the one hand to assess the reduction potential ('what is reachable at all?') and, on the other hand, to be able to realize an 'optimal' method with rigorous error control. In order to reach such targets, the interplay of modern methods from numerical mathematics, stochastics, approximation theory and computer science contains enormous potential.

This was the background for initializing a scientific platform on Model Order Reduction of Parametrized Systems (MoRePaS), also in terms of the website www.morepas.org. MoRePaS stands for a collection of research software, preprints, organization of annual PhD student summer schools and several other activities. In order to intensify the collaboration in this emerging field of research, a conference series on MoRePaS has been initialized:

- 2009: Münster (hosted by the Westfälische Wilhelms-Universität Münster, Germany);

- 2012: Schloss Reisensburg (hosted by Ulm University, Germany);

- 2015: Trieste (hosted by the Scuola Internazionale Superiore di Studi Avanzati, Italy).

This special issue is based upon the scientific outcome of the second international workshop on Model Order Reduction of Parametrized Systems (MoRePaS II), which took place at the Science Center of Ulm University at Schloss Reisensburg (Günzburg, Germany), October 2-5, 2012. The topics of MoRePaS II include:

1. Reduced basis methods (RBM);

2. Proper orthogonal decomposition (POD);

3. Approximation theory related to model reduction;

4. Learning theory and compressed sensing;

5. Stochastic and high-dimensional problems;

6. System-theoretic methods;

7. (Discrete) empirical interpolation ((D)EIM);

8. Reduction of coupled problems;

$4 \quad$ SISSA MathLab, Scuola Internazionale Superiore di Studi Avanzati, Via Bonomea 265, 34136 Trieste, Italy

5 Department of Computational \& Applied Mathematics, Rice University, 6100 Main St, MS-134, Houston, TX 77005, USA

6 Institute for Numerical Mathematics, University of Ulm Helmholtzstr, 20 D-89069 Ulm, Germany 


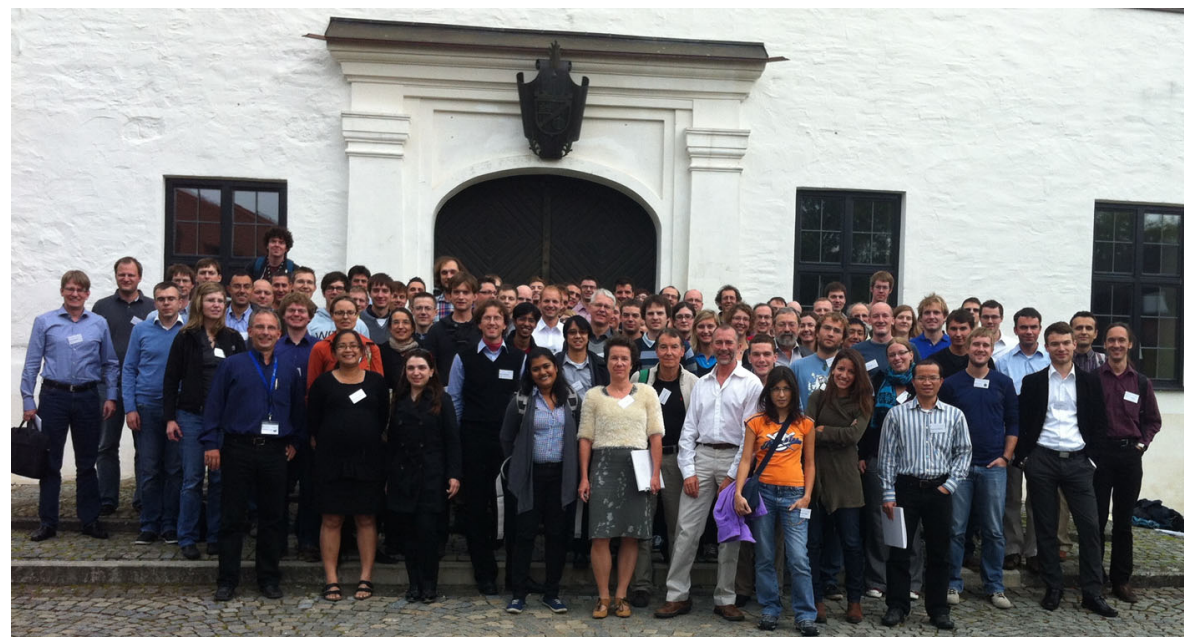

Fig. 1 Participants of MoRePaS II, Science Center of Ulm University at Schloss Reisensburg (Günzburg, Germany), October 2-5, 2012

9. Optimization and optimal control;

10. Krylov-subspace and interpolatory methods.

Those topics have also been used in the call for papers of this special issue. The accepted papers in fact cover most of these areas, only 3. and 4. are not considered deeply in the present collection of papers. It is interesting that most papers origin from a concrete application that serves as a motivation to illustrate mathematical questions of general interest.

The separation of parameters and primitive variables (e.g. space and/or time) is a crucial property (often called affine dependency) for the success of the RBM. As Casenave, Ern and Leliévre point out, the EIM can be used to compute at least an approximative affine decomposition, but this usually requires to access and modify the assembly routines of the corresponding code for the detailed problem, i.e. an intrusive procedure. The authors derive variants of the EIM algorithm that are nonintrusive. Examples of aeroacoustic problems solved by integral equations are shown.

Redeker and Haasdonk are concerned with complex physical models depending on microstructures developing over time. They consider a two-scale model (macroscopic heat equation and a family of microscopic cell problems) describing a phase transition of a binary mixture with the evolution of equiaxed dendritic microstructures. Model reduction is applied to the microscopic model, which is parametrized by the macroscopic temperature yielding to a parameterized, nonlinear, instationary, coupled problem. The method consists of a combination of POD, EIM and a new partitioning approach generating sub-models for different solution regimes.

The contribution by Sommer, Farle and Dyczij-Edlinger introduces a new model reduction method for computing radiation patterns of electrically large antenna arrays over broad frequency bands and wide ranges of look angles. The suggested scheme 
combines finite-element analysis with empirical interpolation and employs a subdomain approach on the Huygens surface to reduce computational costs in the offline part of the algorithm. To assure the reliability of the numerical results, an accurate and efficiently computable a posteriori error bound for the radiation patterns is proposed. A real-world example is presented to demonstrate the efficiency and accuracy of the suggested approach.

The work by Schauer and Linder is devoted to the numerical determination of the electronic structure with the Kohn-Sham equations in combination with the finite element method. The RBM is applied and it is shown that the dimension in allelectron calculations can substantially be reduced. In this context, a generalization of the reduced basis set construction is proposed and shown to increase the flexibility of the method.

Elastic multibody systems (e.g., of cranes, turning processes or gear wheels) are the focus of Fischer and Eberhard with the goal to reduce the elastic degrees of freedom by model order reduction. To gain satisfying reduction results, boundary conditions and acting loads are crucial. However, quite often the boundary conditions or the position of acting loads on the elastic bodies are not known a-priori. In this contribution, the moving load is described by a parameter dependent input matrix, which is the basis for model reduction based on matrix interpolation. Two mechanical examples are examined.

Alla and Volkwein contribute to this special issue with a first mathematically rigorous analysis of the interplay of Proper Orthogonal Decomposition (POD) and Model Predictive Control (MPC). A stabilizing feedback control is computed for a semilinear parabolic partial differential equation utilizing a nonlinear model predictive method. Classical estimates for the model reduction error in POD are combined with the horizon truncation error in MPC. Finally, asymptotic stability of the computed feedback controls is derived for the combined POD-MPC algorithm, based on an appropriate choice of the lengths of the finite time horizons. Numerical experiments are given to confirm the theoretical results.

Cardiac Electrophysiology involving high-dimensional parametric equation systems is the background of the work by Gerbeau, Kombardi and Schenone, who apply a reduced-order method based on Approximated Lax Pairs (ALP). The method is tested on two and three dimensional test-cases, of increasing complexity. The solutions are compared to the ones obtained by a detailed finite element method.

Maier (Martini), Rozza and Haasdonk present a reduced basis method for coupled flow problems modeled in terms of Stokes-Darcy systems, which is based upon a heterogeneous domain decomposition formulation. The paper introduces corresponding a-posteriori bounds that can be evaluated efficiently also in the online stage. The offline phase makes use of the decomposed problem structure. Numerical experiments dealing with groundwater flow scenarios demonstrate the efficiency of the approach.

The contribution by Wieland is devoted to a reduction of the number of terms in an affine decomposition within the RBM. Based upon the EIM, the author generalizes existing partitioning concepts to arbitrary input functions with possibly unknown, high-dimensional, or even without direct parameter dependencies. No a-priori information about the input is necessary. The main idea is to perform several EIMs 
such that different parts of the family of possible input functions are covered. For a new input in the online stage, the coefficients of the resulting affine decomposition are used for the assignment to the appropriate EIM. An application includes parameterized PDEs with stochastic influences.

Any projection-based model reduction method uses a (hopefully) small dimensional subspace on which the full model is projected. Sometimes, a single fixed subspace is not sufficient to reach a desired tolerance. This is the motivation for Amsallem, Zahr and Washabaugh to consider local reduced basis updates for parameterized initial value problems either based on an offline/online decomposition or based upon an offline computed metric acting only on 'relevant' components. An error analysis associated with this approximated metric is then conducted and it is shown that the metric has a kernel interpretation. Two nonlinear physical systems serve as illustrative examples.

The paper by Benner, Grundel and Hornung is concerned with model reduction of parameterized linear time-invariant systems. The parametric dependence is approximated as a so-called metamodel, which allows for the desired model reduction. The authors suggest a metamodel based upon the coefficients of the characteristic polynomial together with $k$-means clustering and radial basis function interpolation. This allows for an accurate and efficient approximation of optimal interpolation points yielding the $\mathcal{H}_{2}$-optimal reduced order model.

Manzoni and Negri present some heuristic strategies to compute rapid and reliable approximations to stability factors (that are a crucial ingredient in error estimators) in nonlinear, inf-sup stable parametrized PDEs. Usually, those factors depend on the unknown detailed solution. As a remedy, the authors propose a linearized, heuristic version of the well-known Successive Constraint Method (SCM), providing a suitable estimate (rather than a rigorous lower bound as in the original SCM) of the stability factor. For the sake of computational efficiency, an alternative heuristic strategy is developed, which combines a radial basis interpolant, suitable criteria to ensure its positiveness, and an adaptive choice of interpolation points through a greedy procedure. Some theoretical results are presented for both schemes accompanied by tests for the Navier-Stokes equations including bifurcation points.

San and Iliescu develop a POD-based reduced-order (POD-ROM) model for midlatitude simplified oceanic basins modeled by the barotropic vorticity equation. Two closure schemes are used to model the effects of the discarded POD modes,namely a mode dependent eddy viscosity closure model and a Smagorinsky-type model. A sensitivity analysis with respect to the free eddy viscosity stabilization parameter is performed. The POD-ROM results are validated against the Munk layer resolving direct numerical simulations. For a four-gyre ocean circulation problem, the new POD-ROM closure models show significant improvements in accuracy over the standard Galerkin model.

Parameterized circuit equations are considered by Son and Stykel. State-of-theart interpolation-based model order reduction methods are analyzed in great detail. This yields a deep mathematical understanding of the considered methods and clearly shows advantages and disadvantages of each method. The considered methods are also numerically tested on two circuit models. 
Himpe and Ohlberger present an accelerated optimization-based approach for combined state and parameter reduction of a parametrized linear control system which is then used as a surrogate model in a Bayesian inverse setting. Following ideas by Lieberman, Willcox and Ghattas (2010), the new approach is based upon a generalized data-driven optimization functional and a Monte-Carlo basis enrichment strategy. The generalization and enhancements presented in this work are shown to decrease overall computational time and increase accuracy of the reduced order model and thus allow an application to large-scale problems. Numerical experiments for a fMRI connectivity model are presented.

The paper by Peherstorfer, Gómez and Bungartz is concerned with pricing of financial products, which can also be seen as a parameterized partial differential equation. When multi-asset basket options with multiple underlying assets are considered, a standard discretization suffers from the curse of dimensionality. Thus, the authors use a sparse grid discretization of the pricing equation (e.g. a Black-Scholes equation, or generalizations). A POD-based reduced order model is derived, which is shown to be applicable to multi-asset options. While preserving sufficient accuracy as compared to the high-fidelity model, speedup factors between 80 and 160 are derived.

The guest editors of this special issue hope that the reader finds an outstanding overview on recent results, developments and methods of MoRePaS. This special issue marks the current leading edge of research and will be a valuable source for researchers working in this field. We thank the editors in chief of Advances in Computational Mathematics (ACOM) for the opportunity to realize this special issue as a collected output of state-of-the-art high-level research in MoRePaS. We thank all authors for their contributions and all anonymous referees for their careful and constructive work. Finally, we are grateful for the ACOM staff for the technical support. 